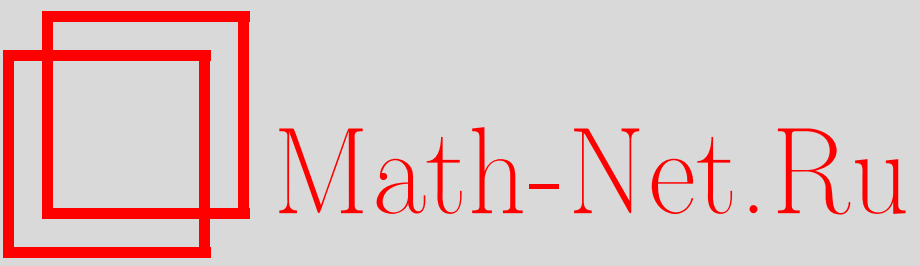

Е. В. Потемкина, Режимы с обострениями в задаче Коши для неоднородного уравнения теплопроводности, УМН, 1996, том 51, выпуск 6, 221-222

DOI: https://doi.org/10.4213/rm1037

Использование Общероссийского математического портала Math-Net.Ru подразумевает, что вы прочитали и согласны с пользовательским соглашением

http://www.mathnet.ru/rus/agreement

Параметры загрузки:

IP: 52.205 .19 .152

26 апреля 2023 г., 15:17:24 


\title{
РЕЖИМЫ С ОБОСТРЕНИЯМИ В ЗАДАЧЕ КОШИ ДЛЯ НЕОДНОРОДНОГО УРАВНЕНИЯ ТЕПЛОПРОВОДНОСТИ
}

\author{
Е.В. ПОТЕМКИНА
}

1. В настоящей работе изучается подобный эффект в случае задачи Коши для неоднородного уравнения теплопроводности с неограниченно возрастающим за конечное время свободным членом. А именно, рассматривается следующая задача:

$$
\begin{gathered}
\partial_{t} u(x, t)=a^{2} \partial_{x}^{2} u(x, t)+f(x, t), \quad x \in \mathbb{R}, \quad t \in(0, T), \\
u(x, 0)=0, \quad x \in \mathbb{R},
\end{gathered}
$$

где $a=$ const $>0$ и $\lim _{t \rightarrow T_{-}} f(x, t)=+\infty$ для $x$ из некоторого подмножества $\mathbb{R}$. При этом предполагается, что:

$$
f(x, t)=\exp \{\omega(t)-\theta(t) g(x)\}
$$

где $\omega(t)$ и $\theta(t)$ - непрерывные положительные функции, $\lim _{t \rightarrow T_{-}} \omega(t)=\lim _{t \rightarrow T_{-}} \theta(t)=+\infty$, $g(x)$ непрерьвна и ограничена снизу. Сначала при общих предположениях относительно $\omega(t)$ и $\theta(t)$ указываются границы тех минимальных множеств, на которых решение неограниченно возрастает или остается ограниченным в течение времени обострения. Затем разбирается частный случай $\omega(t)=m(T-t)^{-\alpha}, \theta(t)=(T-t)^{-\beta}$.

2. Основным результатом данной работы является следующая

Теорема 1. Предположим, что $u(x, t)$ - решение задачи (1)-(3), принадлежащее $C_{x, t}^{2,1}(\mathbb{R} \times[0, T-\varepsilon]) \cap L_{\infty}(\mathbb{R} \times[0, T-\varepsilon]) \quad \forall \varepsilon \in(0, T) ; \omega(t)>0$ u $\theta(t)>0$ принадлежат $C([0, T]), \lim _{t \rightarrow T_{-}} \omega(t)=\lim _{t \rightarrow T_{-}} \theta(t)=+\infty u \omega(t) \geqslant \omega_{1}(T-t)^{-\alpha}, \theta(t) \leqslant \theta_{1}(T-t)^{-\beta}$ при $0 \leqslant t \leqslant T$ для некоторых $\omega_{1}>0, \theta_{1}>0, \alpha>0, \beta>0 ; g(x) \in C(\mathbb{R})$, inf $g(x)>-\infty$. Справедливь приводимье ниже утверждения.

I. Пусть $\lim _{t \rightarrow T_{-}} \omega(t) \theta^{-1}(t)=0, g(x) \geqslant 0, E:=\{x \in \mathbb{R}: g(x)=0\} \neq \varnothing$ u $g(x)$ удовлетворяет условию Гёльдера на Е. Тогда:

a) $е c л u \lim _{t \rightarrow T_{-}} \omega(t)(T-t)^{-1}=0$, mo

$$
\lim _{t \rightarrow T_{-}} u(x, t)=+\infty \quad \forall x \in E, \quad \varlimsup_{t \rightarrow T_{-}} u(x, t)<+\infty \quad \forall x \notin E ;
$$

б) $е c л u \underline{\lim }_{t \rightarrow T_{-}} \omega(t)(T-t)^{-1}=n>0 u \varlimsup_{t \rightarrow T_{-}} \omega(t)(T-t)^{-1}=N<+\infty, m o$

$$
\lim _{t \rightarrow T_{-}} u(x, t)=+\infty \quad \forall x \in E^{*}, \quad \varlimsup_{t \rightarrow T_{-}} u(x, t)<+\infty \quad \forall x \notin E^{* *},
$$

где $E^{*}:=\{x \in \mathbb{R}: \exists \xi \in E(|x-\xi|<2 a \sqrt{n})\}, E^{* *}:=\{x \in \mathbb{R}: \forall \xi \in E(|x-\xi|>2 a \sqrt{N})\} ;$

в) если $\lim _{t \rightarrow T_{-}} \omega(t)(T-t)^{-1}=+\infty, \operatorname{mo~}_{t \rightarrow T_{-}} u(x, t)=+\infty \quad \forall x \in \mathbb{R}$.

II. $\Pi y c m b \underline{\lim }_{t \rightarrow T_{-}} \omega(t) \theta^{-1}(t)=m>0, \varlimsup_{\lim _{t \rightarrow T-}} \omega(t) \theta^{-1}(t)=M<+\infty$,

$G^{*}:=\{x \in \mathbb{R}: g(x)<m\} \neq \varnothing u G^{* *}:=\{x \in \mathbb{R}: g(x)>M\} \neq \varnothing$. Тогдa:

a) ecлu $\lim _{t \rightarrow T_{-}} \omega(t)(T-t)^{-1}=0$, mo

$$
\lim _{t \rightarrow T_{-}} u(x, t)=+\infty \quad \forall x \in G^{*}, \quad \varlimsup_{t \rightarrow T_{-}} u(x, t)<+\infty \quad \forall x \in G^{* *} ;
$$

б) $е с л u \underline{\lim }_{t \rightarrow T_{-}} \omega(t)(T-t)^{-1}=n>0 u \varlimsup_{\lim _{t \rightarrow T_{-}}} \omega(t)(T-t)^{-1}=N<+\infty, m o$

$$
\lim _{t \rightarrow T_{-}} u(x, t)=+\infty \quad \forall x \in G^{\prime}, \quad \varlimsup_{t \rightarrow T_{-}} u(x, t)<+\infty \quad \forall x \in G^{\prime \prime},
$$


əде

$$
\begin{aligned}
G^{\prime} & :=\left\{x \in \mathbb{R}: \exists \xi \in G^{*}\left(|x-\xi| \leqslant 2 a \sqrt{n_{1}(m-g(\xi))}\right)\right\}, \\
G^{\prime \prime}:=\{x \in \mathbb{R}: & \left.\forall \xi \in G^{-}\left(|x-\xi|>2 a \sqrt{N-N_{1} g(\xi)}\right) \forall \xi \in G^{+}\left(|x-\xi|>2 a \sqrt{N-n_{1} g(\xi)}\right)\right\}, \\
& G^{+}:=\{\eta \in \mathbb{R}: g(\eta) \geqslant 0\}, \quad G^{-}:=\{\eta \in \mathbb{R}: g(\eta) \leqslant 0\}, \\
& n_{1}=\varliminf_{t \rightarrow T_{-}} \theta(t)(T-t)^{-1}, \quad N_{1}=\varlimsup_{t \rightarrow T_{-}} \theta(t)(T-t)^{-1} ;
\end{aligned}
$$

в) $е с л и \lim _{t \rightarrow T_{-}} \omega(t)(T-t)^{-1}=+\infty, m o \lim _{t \rightarrow T_{-}} u(x, t)=+\infty \quad \forall x \in \mathbb{R}$.

III. Пусть $\lim _{t \rightarrow T_{-}} \omega(t) \theta^{-1}(t)=+\infty$. Тогдa $\lim _{t \rightarrow T_{-}} u(x, t)=+\infty \quad \forall x \in \mathbb{R}$.

3. Остановимся отдельно на задаче (1), (2) с $f(x, t)=\exp \left\{m(T-t)^{-\alpha}-g(x)(T-t)^{-\beta}\right\}$, где $m>0, \alpha>0, \beta>0, g(x)$ непрерывна и ограничена снизу. Для функций такого вида имеет место

TeOpema 2. I. Пусть $\alpha<\beta, E:=\{x \in \mathbb{R}: g(x)=0\} \neq \varnothing$. Тогдa

a) если $\alpha \in(0,1)$, mo $\lim _{t \rightarrow T_{-}} u(x, t)=+\infty \quad \forall x \in E, \varlimsup_{t \rightarrow T_{-}} u(x, t)<+\infty \quad \forall x \notin E$;

б) если $\alpha=1$, mо $\lim _{t \rightarrow T_{-}} u(x, t)=+\infty \quad \forall x \in E^{*}, \overline{\lim }_{t \rightarrow T_{-}} u(x, t)<+\infty \quad \forall x \notin \bar{E}^{*}$, где $E^{*}:=\{x \in \mathbb{R}: \exists \xi \in E(|x-\xi|<2 a \sqrt{m})\} ;$ для $x \in \partial E^{*}$ в случае, когда существует $\xi^{*} \in \partial E$ maкое, что $\left|x-\xi^{*}\right|=2 a \sqrt{m}$ u $g(\xi) \geqslant c\left|\xi-\xi^{*}\right|^{\gamma}$ при $\xi \rightarrow \xi^{*}, \xi \notin E$, где c $>0$, $\gamma \in(0, \beta]$, справедливо неравенство $\overline{\lim }_{t \rightarrow T_{-}} u(x, t)<+\infty$; в случае, когда для $x \in \partial E^{*}$ существует $\xi^{*} \in \partial E$ такое, что $\left|x-\xi^{*}\right|=2 a \sqrt{m}$ и $g(\xi) \leqslant c\left|\xi-\xi^{*}\right|^{\gamma}$ при $\xi \rightarrow \xi^{*}, \xi \notin E$, где $c>0, \gamma>\beta$, справедливо равенство $\lim _{t \rightarrow T_{-}} u(x, t)=+\infty$;

в) если $\alpha>1$, mo $\lim _{t \rightarrow T_{-}} u(x, t)=+\infty \quad \forall x \in \mathbb{R}$.

II. Пусть $\alpha=\beta, G^{*}:=\{x \in \mathbb{R}: g(x)<m\} \neq \varnothing$. Тогда

a) если $\alpha \in(0,1)$, mo $\lim _{t \rightarrow T_{-}} u(x, t)=+\infty \quad \forall x \in G^{*}, \varlimsup_{t \rightarrow T_{-}} u(x, t)<+\infty \quad \forall x \notin \overline{G^{*}}$; в случае, когда для $x \in \partial G^{*} m=g(\xi) \geqslant c|\xi-x|^{\gamma}$ nри $\xi \rightarrow x, \xi \in G^{*}$, где $c>0$, $\gamma \geqslant 2 \alpha$, справедливо неравенство $\lim _{t \rightarrow T_{-}} u(x, t)<+\infty ;$ в случае, когда для $x \in \partial G^{*}$ $m-g(\xi) \leqslant c|\xi-x|^{\gamma}$ при $\xi \rightarrow x, \xi \in G^{*}$, где $c>0, \gamma \in(0,2 \alpha)$, справедливо равенство $\lim _{t \rightarrow T_{-}} u(x, t)=+\infty$

б) если $\alpha=1$, mо $\lim _{t \rightarrow T_{-}} u(x, t)=+\infty \quad \forall x \in G^{\prime}, \varlimsup_{t \rightarrow T_{-}} u(x, t)<+\infty \quad \forall x \notin G^{\prime}$, əде $G^{\prime}:=\left\{x \in \mathbb{R}: \exists \xi \in G^{*}(|x-\xi| \leqslant 2 a \sqrt{(m-g(\xi))})\right\} ;$

в) если $\alpha>1$, mо $\forall x \in \mathbb{R} \quad \lim _{t \rightarrow T_{-}} u(x, t)=+\infty$.

III. Пусть $\alpha>\beta$. Тогдa $\forall x \in \mathbb{R} \lim _{t \rightarrow T_{-}} u(x, t)=+\infty$.

\section{СПИСОК ЛИТЕРАТУРЫ}

[1] Самарский А.А., Галактионов В.А., Курдюмов С. П., Михайлов А.П. // ДАН СССР. 1979. Т. 247. № 2. С. 349-353. [2] Самарский А. А., Галактионов В. А., Курдюмов С. П., Михайлов А. П. Режимы с обострениями в задачах для квазилинейных параболических уравнений. М.: Наука, 1987. 